\begin{tabular}{|c|c|c|c|c|c|c|}
\hline $\mathrm{n}$ & Age & Sex & BMI & LEPT & IL6 & $\mathrm{PaO2}$ _pre \\
\hline 1 & 47 & $M$ & 45 & 34 & 2,5 & 71,1 \\
\hline 2 & 58 & $\mathrm{~F}$ & 45,5 & 42 & 10,1 & 72,3 \\
\hline 3 & 46 & $\mathrm{~F}$ & 44,4 & 28 & 2,2 & 90 \\
\hline 4 & 41 & $M$ & 39,5 & 29 & 5,9 & 86,3 \\
\hline 6 & 44 & $M$ & 45 & 32 & 2,8 & 84,4 \\
\hline 7 & 57 & $F$ & 40 & 53 & 3 & 86,4 \\
\hline 9 & 51 & $\mathrm{~F}$ & 46,7 & 50 & 4,3 & 78,3 \\
\hline 10 & 52 & $\mathrm{~F}$ & 36,4 & 64 & 3,2 & 96,8 \\
\hline 11 & 58 & $M$ & 49,5 & 13 & 4,3 & 79,8 \\
\hline 12 & 53 & $\mathrm{~F}$ & 54,9 & 88 & 4 & 64,7 \\
\hline 13 & 51 & $M$ & 47,7 & 16 & 3 & 72 \\
\hline 14 & 40 & $\mathrm{~F}$ & 55,1 & 47 & 2,2 & 80 \\
\hline 15 & 40 & $F$ & 47,7 & 59 & 1,5 & 83,5 \\
\hline 16 & 24 & $\mathrm{~F}$ & 45,7 & 64 & 7,5 & 97 \\
\hline 17 & 36 & $F$ & 40,4 & 43 & 1,5 & 94,3 \\
\hline 18 & 29 & $\mathrm{~F}$ & 51,2 & 30 & 3,6 & 76,9 \\
\hline 19 & 43 & $\mathrm{~F}$ & 46,8 & 34 & 2,9 & 82,1 \\
\hline 20 & 41 & $M$ & 40,2 & 24 & 3,6 & 77,8 \\
\hline 21 & 38 & $\mathrm{~F}$ & 44,3 & 64 & 2,4 & 82 \\
\hline 22 & 51 & $F$ & 46,4 & 52 & 4 & 97 \\
\hline 23 & 45 & $M$ & 57,7 & 44 & 3,2 & 84,8 \\
\hline 24 & 56 & $F$ & 49,2 & 29 & 3,4 & 74,7 \\
\hline 25 & 54 & $\mathrm{~F}$ & 35,7 & 42 & 2,7 & 72,3 \\
\hline 26 & 48 & $\mathrm{~F}$ & 41 & 48,4 & 3,8 & 94,9 \\
\hline 27 & 42 & $F$ & 46,8 & 39 & 11,3 & 86,2 \\
\hline 28 & 41 & $F$ & 51,6 & 62 & 3,5 & 73,9 \\
\hline 29 & 59 & $F$ & 41 & 43 & 4,1 & 80,2 \\
\hline 30 & 43 & $M$ & 44,7 & 17 & 1,9 & 70,5 \\
\hline 31 & 49 & $M$ & 43,7 & 28 & 6 & 68,3 \\
\hline 32 & 53 & $M$ & 51,3 & 42 & 4,1 & 73 \\
\hline 33 & 46 & $\mathrm{~F}$ & 39,2 & 35 & 4,2 & 90 \\
\hline 34 & 60 & $F$ & 45,6 & 29 & 4,3 & 75,4 \\
\hline 35 & 37 & $\mathrm{~F}$ & 37,8 & 21 & 3,7 & 78 \\
\hline 36 & 42 & $M$ & 46,9 & 44 & 7,1 & 63,6 \\
\hline 37 & 33 & $\mathrm{~F}$ & 43,1 & 35 & 3,7 & 87,2 \\
\hline 38 & 56 & $\mathrm{~F}$ & 36,5 & 50 & 2,6 & 94,6 \\
\hline 39 & 45 & $M$ & 38,9 & 12 & 3,1 & 84,5 \\
\hline 40 & 48 & $M$ & 41,6 & 17 & 1,5 & 80,3 \\
\hline 41 & 46 & $F$ & 59,6 & 31 & 5,3 & 88,1 \\
\hline 42 & 45 & $F$ & 50,7 & 43 & 3,7 & 84,8 \\
\hline 43 & 52 & $F$ & 43,4 & 48 & 2,5 & 94,4 \\
\hline 44 & 35 & $F$ & 66,2 & 49 & 5,9 & 70,5 \\
\hline 45 & 59 & $\mathrm{~F}$ & 44,8 & 44 & 3,6 & 76,1 \\
\hline 46 & 33 & $\mathrm{~F}$ & 39,5 & 56 & 3,3 & 81,9 \\
\hline
\end{tabular}




\begin{tabular}{|c|c|c|c|c|c|c|}
\hline 47 & 47 & $\mathrm{~F}$ & 46,2 & 48 & 2,3 & 70,5 \\
\hline 48 & 46 & $\mathrm{~F}$ & 41,4 & 41 & 3,8 & 72,5 \\
\hline 49 & 50 & $\mathrm{~F}$ & 40,2 & 47 & 3,3 & 72,3 \\
\hline 50 & 50 & $\mathrm{~F}$ & 52,4 & 53 & 5 & 73,4 \\
\hline 51 & 54 & $F$ & 50,6 & 64 & 6,4 & 87,4 \\
\hline 53 & 48 & $F$ & 49,7 & 27 & 7 & 64 \\
\hline 54 & 66 & $F$ & 35,8 & 38 & 3,6 & 80 \\
\hline 55 & 43 & $\mathrm{~F}$ & 43,7 & 31 & 1,9 & 74,4 \\
\hline 56 & 28 & $\mathrm{~F}$ & 35,6 & 34 & 2,3 & 100,3 \\
\hline 57 & 40 & $F$ & 41 & 17 & 1,5 & 87,4 \\
\hline 58 & 45 & $F$ & 36,2 & 39 & 2,6 & 89,9 \\
\hline 59 & 51 & $F$ & 43,8 & 45 & 3,7 & 82,2 \\
\hline 60 & 52 & $\mathrm{~F}$ & 36,9 & 28 & 1,8 & 76,2 \\
\hline 61 & 49 & $F$ & 38,7 & 49 & 3,9 & 98,9 \\
\hline 63 & 52 & $F$ & 42,2 & 39 & 2,4 & 78,8 \\
\hline 64 & 33 & $\mathrm{~F}$ & 47,6 & 51 & 4,2 & 53,6 \\
\hline 65 & 47 & $F$ & 38,2 & 28 & 1,8 & 86,3 \\
\hline 66 & 44 & $\mathrm{~F}$ & 37 & 31 & 3,1 & 89,5 \\
\hline 68 & 46 & $\mathrm{~F}$ & 45 & 52 & 5,3 & 65,8 \\
\hline 69 & 58 & $M$ & 45 & 19 & 1,7 & 78 \\
\hline 70 & 46 & $\mathrm{~F}$ & 42 & 23 & 1,5 & 76,3 \\
\hline 71 & 36 & $\mathrm{~F}$ & 38 & 34 & 2,9 & 83,1 \\
\hline 72 & 48 & $M$ & 36 & 18 & 1,9 & 86,8 \\
\hline 73 & 37 & $\mathrm{~F}$ & 45 & 39 & 3,4 & 71,2 \\
\hline 74 & 63 & $M$ & 36,3 & 14 & 1,5 & 79,9 \\
\hline 75 & 49 & $F$ & 44 & 42 & 1,5 & 72,5 \\
\hline 76 & 34 & $\mathrm{~F}$ & 41 & 48 & 5,9 & 70,3 \\
\hline 77 & 50 & $\mathrm{~F}$ & 48,5 & 41 & 2,9 & 72,9 \\
\hline 78 & 43 & $\mathrm{~F}$ & 40 & 26 & 2,3 & 84,8 \\
\hline 79 & 30 & $\mathrm{~F}$ & 49,5 & 39 & 2,5 & 68,7 \\
\hline 80 & 39 & $F$ & 62 & 32 & 3,7 & 75,1 \\
\hline 81 & 59 & $\mathrm{~F}$ & 47,5 & 27 & 6,8 & 91,2 \\
\hline 83 & 51 & $F$ & 43 & 64 & 7,7 & 70,4 \\
\hline 84 & 32 & $\mathrm{~F}$ & 53 & 47 & 4,3 & 68,1 \\
\hline 85 & 36 & $M$ & 41 & 38 & 1,8 & 71,9 \\
\hline 86 & 52 & $M$ & 44,6 & 34 & 2,9 & 81,3 \\
\hline 87 & 57 & $\mathrm{~F}$ & 42,3 & 25 & 1,5 & 82,1 \\
\hline 88 & 49 & $F$ & 41,3 & 47 & 5,9 & 61,2 \\
\hline 89 & 68 & $F$ & 45,4 & 39 & 4,4 & 79,6 \\
\hline 90 & 49 & $F$ & 41 & 45 & 4,5 & 71,4 \\
\hline 91 & 46 & $F$ & 47 & 65 & 8,8 & 67,8 \\
\hline 92 & 49 & $\mathrm{~F}$ & 41,5 & 40 & 3,8 & 69,7 \\
\hline 93 & 46 & $M$ & 45,5 & 20 & 2,6 & 85,7 \\
\hline 94 & 46 & $\mathrm{~F}$ & 41,9 & 50 & 5,7 & 79,2 \\
\hline 95 & 54 & $M$ & 54,2 & 48 & 7,4 & 71,8 \\
\hline
\end{tabular}




$\begin{array}{ccccccc}96 & 43 & M & 47 & 20 & 2,1 & 87,1 \\ 97 & 46 & M & 43,4 & 33 & 2,7 & 71,2 \\ 98 & 47 & M & 50,9 & 18 & 2,9 & 88,9 \\ 99 & 48 & M & 47,8 & 48 & 6,9 & 68,6 \\ 100 & 47 & M & 40 & 43 & 5,3 & 71,9 \\ 101 & 61 & M & 39,3 & 14 & 2,2 & 89,9 \\ 102 & 49 & M & 45,1 & 21 & 3,1 & 73,6 \\ 103 & 44 & M & 50,8 & 48 & 4,3 & 68,9 \\ 104 & 52 & M & 44,6 & 44 & 3,9 & 74,8 \\ 105 & 42 & M & 51,6 & 41 & 2,9 & 72,6 \\ 106 & 46 & M & 45,6 & 20 & 2,6 & 82,3 \\ 107 & 47 & M & 46,9 & 53 & 3,9 & 67,7 \\ 108 & 53 & M & 48,6 & 49 & 4 & 69,8 \\ 109 & 30 & M & 45,2 & 40 & 3,9 & 76,8 \\ 110 & 53 & M & 47,8 & 54 & 5,6 & 69,5 \\ 111 & 54 & M & 50,5 & 36 & 3,8 & 71,7 \\ 112 & 38 & M & 51,2 & 48 & 9,3 & 66,3 \\ 113 & 55 & M & 34,7 & 14 & 1,5 & 88,9 \\ 114 & 49 & M & 47,7 & 22 & 3,4 & 85,9 \\ 115 & 45 & M & 46,6 & 12 & 1,5 & 84,5 \\ 116 & 45 & M & 42,8 & 40 & 1,9 & 71 \\ 117 & 54 & M & 43,8 & 41 & 2,5 & 73,6 \\ 118 & 56 & M & 35,8 & 17 & 1,6 & 84,1 \\ & 45 & & & & & \end{array}$




\begin{tabular}{|c|c|c|c|c|c|}
\hline PaCO2_Pre & PaO2_post & PaCO2_Post & Delta_PaO2 & Delta_PaCo2 & LEPT_div \\
\hline 38,3 & 69 & 35,5 & $-2,1$ & $-2,8$ & 0 \\
\hline 44,3 & 112,2 & 26,8 & 39,9 & $-17,5$ & 1 \\
\hline 32,6 & 84,8 & 43,7 & $-5,2$ & 11,1 & 0 \\
\hline 32,6 & 78 & 40 & $-8,3$ & 7,4 & 0 \\
\hline 35,1 & 74,5 & 45,5 & $-9,9$ & 10,4 & 0 \\
\hline 51,8 & 80,4 & 48,4 & -6 & $-3,4$ & 1 \\
\hline 37,8 & 88,3 & 40,3 & 10 & 2,5 & 1 \\
\hline 36 & 70,4 & 43 & $-26,4$ & 7 & 1 \\
\hline 36,9 & 81,9 & 36,9 & 2,1 & 0 & 0 \\
\hline 39,7 & 72,3 & 48,8 & 7,6 & 9,1 & 1 \\
\hline 46,1 & 55,7 & 47,1 & $-16,3$ & 1 & 0 \\
\hline 39 & 79,5 & 37,9 & $-0,5$ & $-1,1$ & 1 \\
\hline 34,4 & 88,1 & 32 & 4,6 & $-2,4$ & 1 \\
\hline 34,1 & 93,4 & 41,9 & $-3,6$ & 7,8 & 1 \\
\hline 29,3 & 83,3 & 39,4 & -11 & 10,1 & 1 \\
\hline 34 & 85,9 & 40,3 & 9 & 6,3 & 0 \\
\hline 33,6 & 86,2 & 37 & 4,1 & 3,4 & 0 \\
\hline 32,8 & 92,8 & 33,5 & 15 & 0,7 & 0 \\
\hline 30,8 & 108,8 & 36,5 & 26,8 & 5,7 & 1 \\
\hline 34,1 & 93,4 & 41,9 & $-3,6$ & 7,8 & 1 \\
\hline 35,4 & 91,8 & 36,5 & 7 & 1,1 & 1 \\
\hline 41 & 69,4 & 31,2 & $-5,3$ & $-9,8$ & 0 \\
\hline 38 & 73,4 & 45,7 & 1,1 & 7,7 & 1 \\
\hline 33,4 & 89,8 & 37,1 & $-5,1$ & 3,7 & 1 \\
\hline 37,8 & 73,2 & 42,2 & -13 & 4,4 & 0 \\
\hline 32,5 & 91,2 & 38,8 & 17,3 & 6,3 & 1 \\
\hline 30,7 & 77,9 & 29,8 & $-2,3$ & $-0,9$ & 1 \\
\hline 41,7 & 79 & 41,4 & 8,5 & $-0,3$ & 0 \\
\hline 41,1 & 63,6 & 44,9 & $-4,7$ & 3,8 & 0 \\
\hline 38,4 & 79,9 & 38,9 & 6,9 & 0,5 & 1 \\
\hline 32,6 & 84,8 & 43,7 & $-5,2$ & 11,1 & 0 \\
\hline 33,8 & 102,9 & 29,8 & 25,5 & -4 & 0 \\
\hline 35,6 & 87 & 35,1 & 9 & $-0,5$ & 0 \\
\hline 38,8 & 61 & 48,7 & $-2,6$ & 9,9 & 1 \\
\hline 31,8 & 79,1 & 39,7 & $-8,1$ & 7,9 & 0 \\
\hline 36,7 & 82,9 & 46,4 & $-11,7$ & 9,7 & 1 \\
\hline 32,2 & 70,9 & 38,8 & $-13,6$ & 6,6 & 0 \\
\hline 37,1 & 83 & 41,8 & 2,7 & 4,7 & 0 \\
\hline 35,6 & 82,2 & 42,7 & $-5,9$ & 7,1 & 0 \\
\hline 35,4 & 91,8 & 36,5 & 7 & 1,1 & 1 \\
\hline 36,4 & 88,5 & 42 & $-5,9$ & 5,6 & 1 \\
\hline 41,7 & 79 & 41,4 & 8,5 & $-0,3$ & 1 \\
\hline 37,1 & 100,4 & 41,9 & 24,3 & 4,8 & 1 \\
\hline 33,7 & 108,8 & 23,2 & 26,9 & $-10,5$ & 1 \\
\hline
\end{tabular}




\begin{tabular}{|c|c|c|c|c|c|}
\hline 41,7 & 79 & 41,4 & 8,5 & $-0,3$ & 1 \\
\hline 34,9 & 67,4 & 43 & $-5,1$ & 8,1 & 1 \\
\hline 44,3 & 112,2 & 26,8 & 39,9 & $-17,5$ & 1 \\
\hline 34 & 74 & 42,9 & 0,6 & 8,9 & 1 \\
\hline 33,5 & 97,7 & 24,1 & 10,3 & $-9,4$ & 1 \\
\hline 35,5 & 61,4 & 43 & $-2,6$ & 7,5 & 0 \\
\hline 30,4 & 102,8 & 33 & 22,8 & 2,6 & 0 \\
\hline 40,8 & 83,2 & 31,9 & 8,8 & $-8,9$ & 0 \\
\hline 24,7 & 88,8 & 37 & $-11,5$ & 12,3 & 0 \\
\hline 32,5 & 69,1 & 38,9 & $-18,3$ & 6,4 & 0 \\
\hline 31,5 & 91,4 & 44,6 & 1,5 & 13,1 & 0 \\
\hline 42,8 & 75,6 & 40,9 & $-6,6$ & $-1,9$ & 1 \\
\hline 36 & 69,5 & 49,7 & $-6,7$ & 13,7 & 0 \\
\hline 29 & 88,9 & 35 & -10 & 6 & 1 \\
\hline 40 & 69,2 & 49,2 & $-9,6$ & 9,2 & 0 \\
\hline 40,1 & 82,3 & 44,1 & 28,7 & 4 & 1 \\
\hline 36,4 & 82,2 & 25,7 & $-4,1$ & $-10,7$ & 0 \\
\hline 34,2 & 88,9 & 41,4 & 0,6 & 7,2 & 0 \\
\hline 35,6 & 64,4 & 40,8 & $-1,4$ & 5,2 & 1 \\
\hline 45,6 & 77,2 & 43,8 & $-0,8$ & $-1,8$ & 0 \\
\hline 42,1 & 79,1 & 43,2 & 2,8 & 1,1 & 0 \\
\hline 33,4 & 76,5 & 39,9 & $-6,6$ & 6,5 & 0 \\
\hline 38 & 72,8 & 39,4 & -14 & 1,4 & 0 \\
\hline 38,9 & 79,9 & 36,7 & 8,7 & $-2,2$ & 0 \\
\hline 38,7 & 78,7 & 38,7 & $-1,2$ & 0 & 0 \\
\hline 36,9 & 77,2 & 39,2 & 4,7 & 2,3 & 1 \\
\hline 38,8 & 74,4 & 40,6 & 4,1 & 1,8 & 1 \\
\hline 40,6 & 86,5 & 37,6 & 13,6 & -3 & 1 \\
\hline 41,1 & 83 & 42,6 & $-1,8$ & 1,5 & 0 \\
\hline 42,4 & 66,1 & 43,2 & $-2,6$ & 0,8 & 0 \\
\hline 38,9 & 91,2 & 40,2 & 16,1 & 1,3 & 0 \\
\hline 40,2 & 92,7 & 39,9 & 1,5 & $-0,3$ & 0 \\
\hline 41,5 & 86 & 43,1 & 15,6 & 1,6 & 1 \\
\hline 42,3 & 81,9 & 46,2 & 13,8 & 3,9 & 1 \\
\hline 43,2 & 67,1 & 39,6 & $-4,8$ & $-3,6$ & 0 \\
\hline 38,8 & 75,1 & 39,7 & $-6,2$ & 0,9 & 0 \\
\hline 41,2 & 73 & 42,4 & $-9,1$ & 1,2 & 0 \\
\hline 40,3 & 66,7 & 34,8 & 5,5 & $-5,5$ & 1 \\
\hline 47 & 51,4 & 50,1 & $-28,2$ & 3,1 & 0 \\
\hline 41,1 & 83,5 & 39,3 & 12,1 & $-1,8$ & 1 \\
\hline 37,9 & 77,9 & 35,8 & 10,1 & $-2,1$ & 1 \\
\hline 40,1 & 71,1 & 39,8 & 1,4 & $-0,3$ & 1 \\
\hline 42,4 & 83 & 47,6 & $-2,7$ & 5,2 & 0 \\
\hline 39,1 & 85,1 & 36,1 & 5,9 & -3 & 1 \\
\hline 40,2 & 79,4 & 38,7 & 7,6 & $-1,5$ & 1 \\
\hline
\end{tabular}




$\begin{array}{cccccc}36,5 & 89,8 & 38,2 & 2,7 & 1,7 & 0 \\ 36,3 & 72,6 & 39,4 & 1,4 & 3,1 & 0 \\ 39,6 & 92,9 & 41,8 & 4 & 2,2 & 0 \\ 38,7 & 79,7 & 36,5 & 11,1 & -2,2 & 1 \\ 38,6 & 83,5 & 37,2 & 11,6 & -1,4 & 1 \\ 39,7 & 87,1 & 41,1 & -2,8 & 1,4 & 0 \\ 41,2 & 68,9 & 42,9 & -4,7 & 1,7 & 0 \\ 38,8 & 78,3 & 35,7 & 9,4 & -3,1 & 1 \\ 37,4 & 81,8 & 36,5 & 7 & -0,9 & 1 \\ 42,3 & 77,9 & 35,9 & 5,3 & -6,4 & 1 \\ 41,3 & 77,5 & 43,4 & -4,8 & 2,1 & 0 \\ 42,1 & 87,1 & 37,9 & 19,4 & -4,2 & 1 \\ 40,6 & 72,4 & 38,8 & 2,6 & -1,8 & 1 \\ 40 & 69,2 & 44,2 & -7,6 & 4,2 & 1 \\ 40,8 & 66,6 & 36,5 & -2,9 & -4,3 & 1 \\ 38,1 & 65,6 & 41,7 & -6,1 & 3,6 & 0 \\ 41,3 & 72,3 & 44,2 & 6 & 2,9 & 1 \\ 35,6 & 92,4 & 43,4 & 3,5 & 7,8 & 0 \\ 36,2 & 78,9 & 41,3 & -7 & 5,1 & 0 \\ 36,7 & 74,4 & 41,8 & -10,1 & 5,1 & 0 \\ 43,6 & 78,2 & 42,8 & 7,2 & -0,8 & 1 \\ 38,1 & 71,9 & 42,3 & -1,7 & 4,2 & 1 \\ 34,3 & 78,5 & 38,9 & -5,6 & 4,6 & 0\end{array}$




\section{Delta_PaO2 DIV}

$\begin{array}{ll}0 & 0 \\ 1 & 0 \\ 0 & 1 \\ 0 & 1 \\ 0 & 1 \\ 0 & 0\end{array}$

Delta_PaCo2 DIV

1

$\begin{array}{ll}1 & 1\end{array}$

1 1

$1 \quad 1$

$0 \quad 1$

$0 \quad 0$

$1 \quad 0$

0 1

0 1

1 1

1 1

1 1

1 1

0 1

1 1

$0 \quad 0$

11

0 1

$0 \quad 1$

1 1

$0 \quad 0$

$1 \quad 0$

$\begin{array}{ll}1 & 1\end{array}$

11

0 1

1 0

$1 \quad 0$

$\begin{array}{ll}0 & 1\end{array}$

$0 \quad 1$

$\begin{array}{ll}0 & 1\end{array}$

$0 \quad 1$

$\begin{array}{ll}0 & 1\end{array}$

$0 \quad 1$

$1 \quad 1$

$0 \quad 1$

$1 \quad 0$

$1 \quad 1$

10




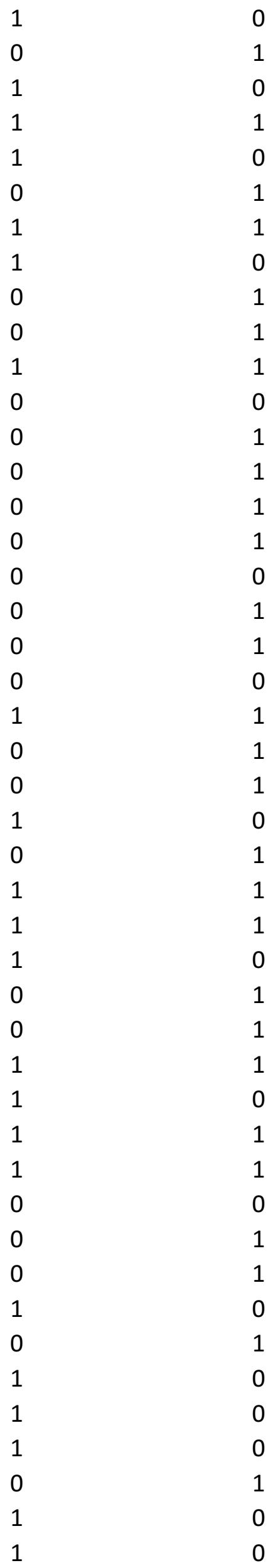




$\begin{array}{ll}1 & 1 \\ 1 & 1 \\ 1 & 1 \\ 1 & 0 \\ 1 & 0 \\ 0 & 1 \\ 0 & 1 \\ 1 & 0 \\ 1 & 0 \\ 1 & 0 \\ 0 & 1 \\ 1 & 0 \\ 1 & 0 \\ 0 & 1 \\ 0 & 0 \\ 0 & 1 \\ 1 & 1 \\ 1 & 1 \\ 0 & 1 \\ 0 & 1 \\ 1 & 0 \\ 0 & 1 \\ 0 & 1\end{array}$

